\title{
Breit einsatzbarer, gut etablierter Integraseinhibitor
}

_ Für PD Christian Hoffmann, niedergelassener Facharzt für Innere Medizin im Infektionsmedizinischen Centrum

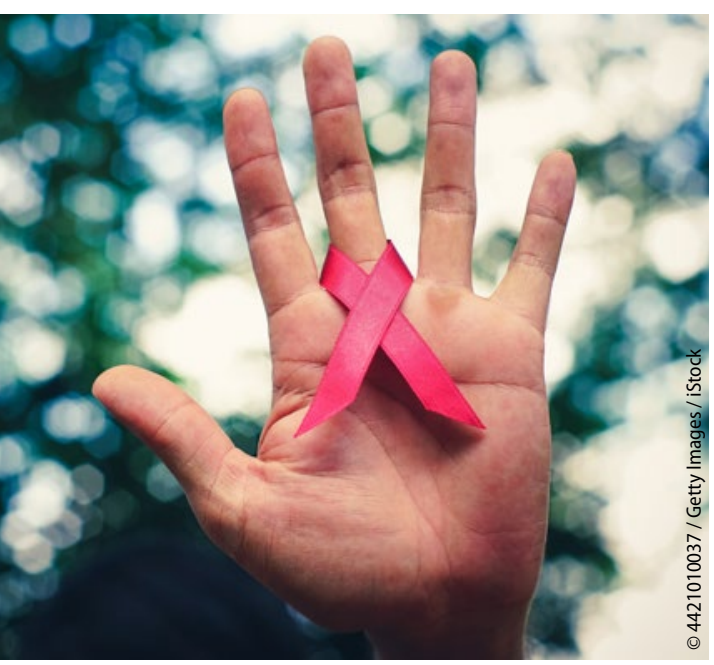

HI-Viren in Schach halten - das klappt heute meistens gut.
Hamburg, bietet sich Raltegravir (Isentress $^{\circledast}$ ) für ein breites Patientenspektrum als Therapieoption an. Er stellte mehrere Fallbeispiele vor, darunter einen HIVPatienten mit einem Lymphom-Rezidiv, einen langjährig vorbehandelten Patienten, der eine Salvagetherapie benötigte, einen sogenannten „Late Presenter“, der sich erst in einem fortgeschrittenen Stadium vorstellte, und einen Patienten mit multiresistenten Viren.

Raltegravir benötigt keine Boosterung und zeichnet sich in Langzeitstudien neben der guten Wirksamkeit und Verträglichkeit auch durch das geringe Potenzial für Wechselwirkungen mit anderen Arzneimitteln aus. Die Substanz wird nicht über das Cytochrom-P450-System, sondern hauptsächlich über die UDP-Glukuronosyltransferase 1A1 metabolisiert.
Dr. Nazifa Qurishi, niedergelassene Internistin in Köln, verwies auf die fehlenden klinisch relevanten Interaktionen zwischen Raltegravir und HepatitisC-Medikamenten. Sie empfahl die Internetseite www.hep-druginteractions.org der University of Liverpool als Wegweiser für die zahlreichen Interaktionen zwischen HIV- und Hepatitis-C-Medikamenten. Demnach könne z. B. die chronische Hepatitis-C-Virus(HCV)Infektion eines HIV-HCV-koinfizierten Patienten unter einer Raltegravir-basierten HIV-Therapie bei Vorliegen eines HCV-Genotyps 1 oder 4 mit Elbasvir/ Grazoprevir (Zepatier ${ }^{\circledR}$ ) behandelt werden, ohne dass relevante Interaktionen auftreten, so Qurishi.

Andrea Warpakowski

- Symposium „Der Patient im Fokus!", 7. Münchner AIDS- und Hepatitis-Werkstatt; München, März 2017 (Veranstalter: MSD)

\section{Antikoagulation bei Vorhofflimmern}

\section{Neue Daten bestätigen niedriges Blutungsrisiko}

_ Das nicht-Vitamin-K-abhängige orale Antikoagulans Apixaban (Eliquis ${ }^{\circledR}$ ) ist im klinischen Versorgungsalltag besser verträglich als die Vitamin-K-Antagonisten Warfarin bzw. Phenprocoumon. Das zeigen Versorgungsstudien aus den USA und jetzt auch aus Deutschland.

Durch die große Patientenzahl und meist lange Beobachtungsdauer können Studien aus dem Versorgungsalltag dazu beitragen, ergänzende Aussagen zur Effektivität und Verträglichkeit eines Medikaments im Behandlungsalltag zu gewinnen. In einer retrospektiven Analyse aus dem US-amerikanischen Versorgungsalltag [Yao X et al. Effectiveness and safety of dabigatran, rivaroxaban, and apixaban versus warfarin in nonvalvular atrial fibrillation. J Am Heart Assoc. 2016;5:e003725] wurden Daten von Pati- enten mit nicht-valvulärem Vorhofflimmern (VHF) ausgewertet. Die Analyse von über 15.000 Patienten der Vergleichskohorte Apixaban vs. Warfarin zeigte, dass unter Apixaban ein signifikant geringeres Risiko für einen Schlaganfall oder eine systemische Embolie im Vergleich zu Warfarin bestand (Hazard Ratio $[\mathrm{HR}] 0,67 ; \mathrm{p}=0,04)$, insbesondere in Bezug auf hämorrhagische Infarkte. Auch das Risiko für schwere Blutungen war signifikant geringer. Diese Daten ergänzen die positiven Ergebnisse kontrollierter Studien zu Apixaban.

\section{Jetzt auch Vergleich zu Phenprocoumon}

Mit der retrospektiven Analyse CARBOS [Hohnloser SH et al. Risk of bleeding with non-vitamin $\mathrm{K}$ antagonists and phenpro- coumon in routine care patients with non-valvular atrial fibrillation. ESC-Kongress 2017, Poster P2608] gibt es nun eine Auswertung, in der Apixaban mit Phenprocoumon verglichen wurde. Sie basiert auf anonymisierten Krankenversicherungsdaten von Patienten mit nicht-valvulärem VHF. Demnach könnte eine Behandlung mit Apixaban $(\mathrm{n}=3.633)$ gegenüber Phenprocoumon $(n=16.179)$ mit einem niedrigeren Risiko für schwere Blutungen (adjustierte HR 0,68; p = 0,008 ), gastrointestinale Blutungen (adjustierte HR 0,53; p < 0,001) sowie Blutungen jeglicher Art (adjustierte HR 0,80; $p=0,002)$ assoziiert sein.

\section{Dr. Beate Grübler}

- Symposium "Standortbestimmungen:Schlaganfallversorgung - Gerinnungsmanagement - NOAKs im Praxisalltag", ANIM 2017; Wien, Februar 2017 (Veranstalter: BMS und Pfizer) 